\title{
Accuracy of blood-glucose measurements using glucose meters and arterial blood gas analyzers in critically ill adult patients: systematic review
}

\author{
Shigeaki Inoue ${ }^{1}$, Moritoki Egi $i^{2 *}$ Joji Kotani ${ }^{3}$ and Kiyoshi Morita ${ }^{2 *}$
}

\begin{abstract}
Introduction: Glucose control to prevent both hyperglycemia and hypoglycemia is important in an intensive care unit. Arterial blood gas analyzers and glucose meters are commonly used to measure blood-glucose concentration in an intensive care unit; however, their accuracies are still unclear.
\end{abstract}

Methods: We performed a systematic literature search (January 1, 2001, to August 31, 2012) to find clinical studies comparing blood-glucose values measured with glucose meters and/or arterial blood gas analyzers with those simultaneously measured with a central laboratory machine in critically ill adult patients.

Results: We reviewed 879 articles and found 21 studies in which the accuracy of blood-glucose monitoring by arterial blood gas analyzers and/or glucometers by using central laboratory methods as references was assessed in critically ill adult patients. Of those 21 studies, 11 studies in which International Organization for Standardization criteria, error-grid method, or percentage of values within $20 \%$ of the error of a reference were used were selected for evaluation. The accuracy of blood-glucose measurements by arterial blood gas analyzers and glucose meters by using arterial blood was significantly higher than that of measurements with glucose meters by using capillary blood (odds ratios for error: 0.04, $P<0.001$; and 0.36, $P<0.001$ ). The accuracy of blood-glucose measurements with arterial blood gas analyzers tended to be higher than that of measurements with glucose meters by using arterial blood $(P=0.20)$. In the hypoglycemic range (defined as $<81 \mathrm{mg} / \mathrm{dl}$ ), the incidence of errors using these devices was higher than that in the nonhypoglycemic range (odds ratios for error: arterial blood gas analyzers, 1.86, $P=0.15$; glucose meters with capillary blood, $1.84, P=0.03$; glucose meters with arterial blood, 2.33, $P=0.02$ ). Unstable hemodynamics (edema and use of a vasopressor) and use of insulin were associated with increased error of blood glucose monitoring with glucose meters.

Conclusions: Our literature review showed that the accuracy of blood-glucose measurements with arterial blood gas analyzers was significantly higher than that of measurements with glucose meters by using capillary blood and tended to be higher than that of measurements with glucose meters by using arterial blood. These results should be interpreted with caution because of the large variation of accuracy among devices. Because blood-glucose monitoring was less accurate within or near the hypoglycemic range, especially in patients with unstable hemodynamics or receiving insulin infusion, we should be aware that current blood glucose-monitoring technology has not reached a high enough degree of accuracy and reliability to lead to appropriate glucose control in critically ill patients.

\footnotetext{
* Correspondence: moriori@tg8.so-net.ne.jp; k_morita@cc.okayama-u.ac.jp ${ }^{2}$ Department of Anesthesiology and Resuscitology, Okayama University

Hospital, 2-5-1 Shikatachou, Okayama, Okayama 700-8525, Japan

Full list of author information is available at the end of the article
} 


\section{Introduction}

Glucose control to prevent both hyperglycemia and hypoglycemia is important in an intensive care unit [1]. Recent meta-analysis, including results of the NICE-SUGAR study [2], showed that intensive insulin therapy (target blood-glucose control, 80 to $110 \mathrm{mg} / \mathrm{dl}$ ) was not beneficial and increased the risk of severe hypoglycemia in critically ill patients [3-5]. Thus, it is currently recommended that insulin should be used when the glucose concentration exceeds $180 \mathrm{mg} / \mathrm{dl}$, and target glucose concentration should generally be between 144 and $180 \mathrm{mg} / \mathrm{dl}$ [6,7]

Even though a more-modest target for blood-glucose concentration is now accepted, the importance of glucose monitoring and its accuracy has become clearer. Because the physiological activity of glucose is dependent on its plasma concentration, central laboratory blood-glucose measurement using plasma (Glu-lab) is recommended $[8,9]$. However, arterial blood gas analyzers (ABGs) and/or glucose meters, not Glu-lab, are commonly used to measure blood-glucose concentrations in critically ill patients, because of their convenience and speed [10]. Because most of these devices were not developed to guide the administration of insulin in critically ill patients, they might not be sufficiently accurate to guide therapy aimed at maintaining blood glucose within a $30-\mathrm{mg} / \mathrm{dl}$ range [11]. Therefore, knowledge of their limitations is essential to minimize the possibility of a harmful measurement error. However, no systematic literature review has assessed the agreement of measurements by ABGs and/or glucose meters in critically ill patients.

Accordingly, we performed a systematic review and meta-analysis of selected observational studies on the accuracy of blood-glucose measurements by using ABGs (Glu-ABGs), glucose meters using capillary blood samples (Gluco-C), and glucose meters using arterial blood samples (Gluco-A) in critically ill adult patients.

\section{Materials and methods}

\section{Electronic database}

We performed a systematic literature search (January 1, 2001, through August 31, 2012) to find clinical studies comparing blood-glucose values measured by using ABGs and/or glucose meters with those simultaneously measured with a central laboratory machine in critically ill adult patients. The literature search was performed by using MEDLINE and PubMed electronic databases with the following key words: "intensive care", "critical care," "glucose," "sugar," "glycemic," "insulin," "Bland Altman," "agreement," "validation," "reliability," "accuracy," "correlation," "Clarke grid," and "bias." All articles identified by this search strategy were obtained, and their bibliographies were studied for articles that might have been missed by the electronic database search.

\section{Inclusion and exclusion criteria}

Inclusion criteria for the current systematic review were as follows: (a) studies conducted in critically ill adult patients, (b) studies in which the accuracy of glucose monitoring was assessed by using ABGs and/or glucose meters, (c) studies in which Glu-lab values were used as reference values, and (d) articles presenting an appropriate summary of statistics. We excluded nonhuman studies, non-English-language articles, and pediatric studies.

\section{Data extraction and interpretation}

Two of the authors (SI and ME) extracted data from selected articles, which were then reviewed by coauthors. We paid particular attention to determine whether the accuracy of blood-glucose monitoring was influenced by types of devices and sites of blood collection. Because the accuracy of blood-glucose monitoring in a hypoglycemic range is important, we performed further assessment of accuracy in a hypoglycemic range, defined as $<81 \mathrm{mg} / \mathrm{dl}$. Additionally, we summarized factors associated with errors of blood-glucose measurements.

\section{Outcomes}

\section{Primary outcome}

Most of the studies were conducted by using (a) agreement (percentages of blood-glucose values with an acceptable error), and/or (b) bias (mean difference between devices and reference) for evaluation.

Because the International Organization for Standardization (ISO) criteria use agreement within $\pm 20 \%$ of Glu-lab at or above $75 \mathrm{mg} / \mathrm{dl}$ and within $\pm 15 \mathrm{mg} / \mathrm{dl}$ below $75 \mathrm{mg} / \mathrm{dl}$, we defined primary outcome as percentages of bloodglucose values within $\pm 20 \%$ of the error of Glu-lab, which involved Zone A of error-grid analysis (agreement within $\pm 20 \%$ of Glu-lab at or above $70 \mathrm{mg} / \mathrm{dl}$ ) and agreement with ISO criteria. We obtained rates of overestimation and underestimation of blood-glucose measurements. We defined proportion of nonagreement $<5 \%$ as good quality of blood-glucose measurements according to ISO criteria.

\section{Secondary outcomes}

We obtained the proportion of agreement by using criteria other than the previously described criteria. Because many reports showed the bias of each device, we summarized their bias.

\section{Statistical analysis}

The current systematic review was performed by following the MOOSE statement for observational studies [12]. Analysis was performed by using Review Manager (RevMan) (The Cochrane Collaboration, 2008; The Nordic Cochrane Centre, Copenhagen, Denmark). Heterogeneity 
was calculated by the I2 test, which shows the rate of variation across studies due to heterogeneity rather than to chance (ranging from 0 (no heterogeneity) to 100 (maximum heterogeneity)) [13]. Given the significant heterogeneity found among the results of the studies, the random-effects model was used [14]. All results are reported with $95 \%$ confidence intervals. A $P$ value $<0.05$ was taken to indicate statistical significance.

\section{Results}

We identified 879 potentially relevant articles by the literature search. We excluded 716 studies because they were animal studies, nonclinical studies, non-Englishlanguage articles, or nonrelated studies. Of the remaining 163 studies, 116 were excluded because they were performed in infant or pediatric populations. Full text reviews were conducted for the remaining 47 articles. In 21 of those 47 studies, the accuracy of blood-glucose monitoring was assessed by using ABGs and/or glucometers with central laboratory methods as references in critically ill adult patients (Figure 1).

Among the 21 selected studies [15-35], 11 studies [15-25] used ISO criteria, the error-grid method, or percentage of values within $20 \%$ of the error of a reference; three studies [26-28] used agreement with criteria other than these criteria, and seven studies [29-35] used only bias for evaluation (Figure 1, Table 1).

Bias of point of blood-glucose monitoring in adult critically ill patients

Bias of point of blood-glucose monitoring in critically ill adult patients was assessed in 20 studies (Table 2). Bias was assessed for Glu-ABGs in five studies [15-18,20], for Gluco-C in 13 studies [18,20-27,29-31,33,34], and for Gluco-A in 12 studies [18,20-23,25,27,28,30-32,35]. The mean differences varied between $-2.7 \mathrm{mg} / \mathrm{dl}$ [17] and

=Electronic literature search=

(sepsis OR septic OR intensive care OR critical care) AND (glucose OR sugar OR glycemic OR insulin) AND (Bland Altman OR agreement OR validation OR reliability OR accuracy OR correlation OR Clarke grid OR bias) (publish data 2000/01/01-2012/8/31)

1

879 potentially relevant studies

716 studies excluded

(animal studies, non-clinical studies, non-English language papers, non-related studies)

116 studies excluded (studies in Infant or pediatric patients)

47 studies for full text review

26 studies excluded (reference was not laboratory blood glucose method, non-critically ill)

21 studies assessed the accuracy of blood glucose monitoring using ABGs and/or glucose meters using central laboratory methods as reference in adult critically ill patients.

- 11 studies using 1) International Organization for Standardization criteria, 2) error grid analysis or 3 ) percentage of values within $20 \%$ of reference value

- 2 studies using percentage of values within $10 \%$ of reference value

- 1 study using percentage of values within $20 \mathrm{mg} / \mathrm{dL}$ difference from reference value

- 7 studies using solely bias for evaluation

Figure 1 Study selection for inclusion systematic review for accuracy of glycemic measurements in the critically ill patients. 
Table 1 Observational studies to test the accuracy of point of blood-glucose monitoring in critically ill adult patients (January 2001 to August 2012)

\begin{tabular}{|c|c|c|c|c|c|c|c|c|c|c|c|c|}
\hline $\begin{array}{l}\text { First author } \\
\text { (year) }\end{array}$ & $\begin{array}{l}\text { Study } \\
\text { design }\end{array}$ & $\begin{array}{c}\text { Age } \\
\text { (years) }\end{array}$ & $\begin{array}{l}\text { APACHE } \\
\text { II }\end{array}$ & No & Protocol & Central Lab Machine & ABG & $\begin{array}{c}\text { Gluco- } \\
\text { C }\end{array}$ & $\begin{array}{c}\text { Gluco- } \\
\text { A }\end{array}$ & $\begin{array}{l}\text { Agreement } \\
\text { (within) }\end{array}$ & Bias & Ref \\
\hline $\begin{array}{l}\text { Stadlbauer V } \\
\text { (2010) }\end{array}$ & Pro & 55 & 17 & 17 & - & Hexokinase method & $\circ$ & - & - & $20 \%$ & Yes & {$[15]$} \\
\hline $\begin{array}{l}\text { Corstjens AM } \\
(2006)\end{array}$ & Pro & $(32-88)$ & - & 45 & $\begin{array}{l}\text { Every } 6 \\
\text { hours }\end{array}$ & YSI2300 & $\circ$ & - & - & $20 \%$ & Yes & [16] \\
\hline $\begin{array}{l}\text { Hoedemaekers CW } \\
\text { (2008) }\end{array}$ & Pro & - & - & 32 & $1 / p t s$ & Aeroset & $\circ$ & - & - & $20 \%$ & Yes & {$[17]$} \\
\hline $\begin{array}{l}\text { Slater-MacLean L } \\
\text { (2008) }\end{array}$ & Pro & 56 & 19 & 60 & 3 per day & YSI2300 & $\circ$ & $\circ$ & $\circ$ & $20 \%$ & Yes & [18] \\
\hline $\begin{array}{l}\text { Kanji S } \\
\text { (2005) }\end{array}$ & Pro & 68 & 22 & 30 & Every 5:00 & $L X-20$ & $\circ$ & $\circ$ & $\circ$ & $20 \%$ & No & [19] \\
\hline $\begin{array}{l}\text { Petersen JR } \\
\text { (2008) }\end{array}$ & Retro & - & - & 84 & $1 / p t s$ & Vitros 950 or $5.1 \mathrm{FS}$ & $\circ$ & $\circ$ & $\circ$ & $20 \%$ & Yes & {$[20]$} \\
\hline $\begin{array}{l}\text { Desachy A } \\
\text { (2008) }\end{array}$ & Pro & 59 & - & 85 & $\begin{array}{l}\text { First } 4 \\
\text { times }\end{array}$ & Dimension Vista device & - & $\circ$ & $\circ$ & $20 \%$ & Yes & {$[21]$} \\
\hline $\begin{array}{l}\text { Pulzi Júnior SA } \\
\text { (2009) }\end{array}$ & Retro & 55 & 16 & 40 & $1 / p t s$ & Au640e & - & $\circ$ & $\circ$ & $20 \%$ & Yes & {$[22]$} \\
\hline $\begin{array}{l}\text { Lonjaret L } \\
\text { (2012) }\end{array}$ & Pro & 59 & - & 75 & Every 5:00 & Glucose oxidase method & - & $\circ$ & $\circ$ & $20 \%$ & Yes & {$[23]$} \\
\hline $\begin{array}{l}\text { Critchell CD } \\
\text { (2007) }\end{array}$ & Pro & 59 & 16 & 80 & $\begin{array}{l}\text { Every } 12 \text { or } \\
24 \mathrm{~h}\end{array}$ & $L X-20$ & - & $\circ$ & - & $20 \%$ & Yes & {$[24]$} \\
\hline $\begin{array}{l}\text { Meynaar IA } \\
\text { (2009) }\end{array}$ & Pro & 72 & 18 & 32 & Every $4 \mathrm{~h}$ & Architect Cl8200 & - & - & $\circ$ & $20 \%$ & Yes & {$[25]$} \\
\hline $\begin{array}{l}\text { Cook A } \\
(2009)\end{array}$ & Pro & 58 & - & 67 & $1 / p t s$ & Au640 & - & $\circ$ & - & $20 \mathrm{mg} / \mathrm{dl}$ & Yes & {$[26]$} \\
\hline $\begin{array}{l}\text { Karon BS } \\
(2007)\end{array}$ & Pro & 69 & - & 20 & $\begin{array}{l}\text { First } 5 \\
\text { hourly }\end{array}$ & Double P Modular system & - & $\circ$ & $\circ$ & $10 \%$ & Yes & {$[27]$} \\
\hline $\begin{array}{l}\text { Karon } \\
\text { (2008) }\end{array}$ & Pro & - & - & - & - & Roche Integra 400 & - & - & $\circ$ & $10 \%$ & Yes & {$[28]$} \\
\hline $\begin{array}{l}\text { Fekih Hassen M } \\
\text { (2010) }\end{array}$ & Pro & 60 & - & 43 & $6 / p t s$ & $\begin{array}{l}\text { Dade-Behring Multichannel } \\
\text { Analyzer }\end{array}$ & - & $\circ$ & - & - & Yes & {$[29]$} \\
\hline $\begin{array}{l}\text { Finkielman JD } \\
\text { (2005) }\end{array}$ & Retro & 56 & - & 197 & - & $\begin{array}{l}\text { Glucose Analyzer } 2 \text { or } \\
\text { Hitachi } 747-200\end{array}$ & - & $\circ$ & $\circ$ & - & Yes & {$[30]$} \\
\hline $\begin{array}{l}\text { Lacala T } \\
(2007)\end{array}$ & Pro & 67 & - & 42 & $1 / \mathrm{pts}$ & $R \times L$ & - & $\circ$ & $\circ$ & - & Yes & {$[31]$} \\
\hline $\begin{array}{l}\text { Mann EA } \\
\text { (2008) }\end{array}$ & Pro & - & - & - & - & Vitros Fusion & - & - & $\circ$ & - & Yes & {$[32]$} \\
\hline $\begin{array}{l}\text { Shearer A } \\
(2009)\end{array}$ & Pro & 64 & - & 63 & $1 / p t s$ & Au640 & - & $\circ$ & - & - & Yes & {$[33]$} \\
\hline $\begin{array}{l}\text { Ray JG } \\
(2001)\end{array}$ & Pro & 67 & - & 10 & - & Cobas Integra Analyzer & - & - & $\circ$ & - & Yes & {$[34]$} \\
\hline $\begin{array}{l}\text { Denfeld QE } \\
\text { (2011) }\end{array}$ & Pro & 61 & - & 46 & $1 / p t s$ & DXC 800 & - & - & $\circ$ & - & Yes & [35] \\
\hline
\end{tabular}

Labo, laboratory; ABG, arterial blood gas analyzer; Gluco-C, glucose meters using capillary blood samples; Gluco-A, glucose meters using arterial blood samples; ${ }^{\text {, }}$ device evaluated, -, not evaluated; pts, patients; Pro, prospective study; Retro, retrospective study.

$25.2 \mathrm{mg} / \mathrm{dl}[16]$ in Glu-ABGs, between $-16 \mathrm{mg} / \mathrm{dl}[23]$ and $9.9 \mathrm{mg} / \mathrm{dl}[22]$ in Gluco-C, and between $-10 \mathrm{mg} / \mathrm{dl}$ [23] and $23.0 \mathrm{mg} / \mathrm{dl}[32]$ in Gluco-A.

Limits of agreements were shown for Glu-ABGs in two reports [15,17], for Gluco-C in 10 studies [21-24,26,29-31, $33,34]$, and for Gluco-A in seven studies [21-23,25,30$32,35]$. Its range (upper limit to lower limit) varied between
$19 \mathrm{mg} / \mathrm{dl} \mathrm{[15]} \mathrm{and} 39 \mathrm{mg} / \mathrm{dl}$ [17] for Glu-ABGs, between $44 \mathrm{mg} / \mathrm{dl}$ [26] and $144 \mathrm{mg} / \mathrm{dl}$ [29] for Gluco-C, and between $38 \mathrm{mg} / \mathrm{dl}$ [32] and $82 \mathrm{mg} / \mathrm{dl}$ [23] for Gluco-A.

\section{Characteristics of 11 inclusion studies}

Eleven studies [15-25] that used ISO criteria, error-grid method, or percentage of values within $20 \%$ of the error of 
Table 2 Bias of point of blood-glucose monitoring in critically ill adult patients

\begin{tabular}{|c|c|c|c|c|c|c|c|}
\hline First author (year) & $\begin{array}{l}\text { Device } \\
\text { (ABG) }\end{array}$ & $\begin{array}{l}\text { Device } \\
\text { (Gluco-C) }\end{array}$ & $\begin{array}{c}\text { Device } \\
\text { (Gluco-A) }\end{array}$ & $\begin{array}{l}\text { Bias } \\
\text { (ABG) }\end{array}$ & $\begin{array}{c}\text { Bias } \\
\text { (Gluco-C) }\end{array}$ & $\begin{array}{c}\text { Bias } \\
\text { (Gluco-A) }\end{array}$ & Ref \\
\hline $\begin{array}{l}\text { Stadlbauer V } \\
\text { (2010) }\end{array}$ & $\begin{array}{c}\text { Cobas B } 221 \\
\text { ABL } 800 \\
\text { Grem Premiere } 3000\end{array}$ & - & - & $\begin{array}{l}8.4(-5.3,22.1) \\
7.6(-1.9,17.0) \\
4.6(-7.9,17.0)\end{array}$ & - & - & [15] \\
\hline $\begin{array}{l}\text { Corstjens AM } \\
\text { (2006) }\end{array}$ & ABL715 & - & - & Mean $\mathrm{D}=25.2$ & - & - & [16] \\
\hline $\begin{array}{l}\text { Hoedemaekers CW } \\
\text { (2008) }\end{array}$ & Rapidlab & - & - & $-2.7(-22.3,16.9)$ & - & - & {$[17]$} \\
\hline $\begin{array}{l}\text { Slater-MacLean L } \\
\text { (2008) }\end{array}$ & Chiron865 & $\begin{array}{c}\text { SureStepflexx } \\
\text { Accu-Chek Inform } \\
\text { FreeStyle } \\
\end{array}$ & $\begin{array}{c}\text { SureStepflexx } \\
\text { Accu-Chek Inform } \\
\text { FreeStyle }\end{array}$ & Mean $D=0.4$ & $\begin{array}{l}\text { Mean D }=9.2 \\
\text { Mean D }=-4.5 \\
\text { Mean D }=5.8\end{array}$ & $\begin{array}{c}\text { Mean } D=3.4 \\
\text { Mean } D=-10.1 \\
\text { Mean } D=1.6\end{array}$ & [18] \\
\hline $\begin{array}{l}\text { Kanji S } \\
(2005)\end{array}$ & RapidLab860 & Accu-Chek Inform & Accu-Chek Inform & - & - & - & [19] \\
\hline $\begin{array}{l}\text { Petersen JR } \\
\text { (2008) }\end{array}$ & Rapidpoint405 & Accu-Chek Inform & Accu-Chek Inform & Mean $\mathrm{D}=1.8$ & Mean $D=9$ & Mean $D=12.6$ & {$[20]$} \\
\hline $\begin{array}{l}\text { Desachy A } \\
\text { (2008) }\end{array}$ & - & Accu-Chek Sensor & Accu-Chek Sensor & - & $1.5(-55.3,58.3)$ & $1.4(-39.5,42.4)$ & {$[21]$} \\
\hline $\begin{array}{l}\text { PulziJúnior SA } \\
\text { (2009) }\end{array}$ & - & FreeStyle & FreeStyle & - & $9.9(-52.4,72.1)$ & $6.8(-30.6 .44 .1)$ & {$[22]$} \\
\hline $\begin{array}{l}\text { Lonjaret L } \\
\text { (2012) }\end{array}$ & - & Contour & Contour & - & $-16(-59.1,27.1)$ & $-10(-51.2,31.2)$ & {$[23]$} \\
\hline $\begin{array}{l}\text { Critchell CD } \\
\text { (2007) }\end{array}$ & - & Accu-Chek Inform & - & - & $8.6(-28.6,45.8)$ & - & {$[24]$} \\
\hline $\begin{array}{l}\text { Meynaar IA } \\
(2009)\end{array}$ & - & - & Accu-Chek Inform & - & - & $11(-20.2,42.2)$ & [25] \\
\hline $\begin{array}{l}\text { Cook A } \\
(2009)\end{array}$ & - & SuperStepFlexx & - & - & $9.5(-12.5,31.5)$ & - & [26] \\
\hline $\begin{array}{l}\text { Karon BS } \\
(2007)\end{array}$ & - & Accu-Chek Inform & Accu-Chek Inform & - & $\begin{array}{c}\text { Median }=-1 \\
{[\text { IQR }-4,5]}\end{array}$ & $\begin{array}{c}\text { Median }=14 \\
{[\text { IQR 10, 18] }}\end{array}$ & {$[27]$} \\
\hline $\begin{array}{l}\text { Karon } \\
\text { (2008) }\end{array}$ & - & - & $\begin{array}{c}\text { Accu-Chek Inform } \\
\text { Precision PCx } \\
\text { SureStepFlexx } \\
\text { StatStrip }\end{array}$ & - & - & $\begin{array}{c}\text { Median }=-9 \\
\text { Median }=-12 \\
\text { Median }=2 \\
\text { Median }=-3\end{array}$ & [28] \\
\hline $\begin{array}{l}\text { Fekih Hassen M } \\
(2010)\end{array}$ & - & Accu-Chek & - & - & $-0.9(-74.3,72.5)$ & - & [29] \\
\hline $\begin{array}{l}\text { Finkielman JD } \\
\text { (2005) }\end{array}$ & - & SureStepFlexx & SureStepFlexx & - & $7.9(-27.2,43.1)^{a}$ & $7.9(-27.2,43.1)^{a}$ & {$[30]$} \\
\hline $\begin{array}{l}\text { Lacala T } \\
\text { (2007) }\end{array}$ & - & Sure Step pro & Sure Step Pro & - & $1.0(-23.1,25.1)$ & $-0.1(-21.7,21.5)$ & {$[31]$} \\
\hline $\begin{array}{l}\text { Mann EA } \\
\text { (2008) }\end{array}$ & - & - & $\begin{array}{c}\text { Sure Step Flexx } \\
\text { Accu-Chek Inform } \\
\text { Accu-Chek Advantage } \\
\text { Precision PCx }\end{array}$ & - & - & $\begin{array}{l}19.1(3.7,34.5) \\
20.7(-0.8,42.2) \\
22.0(-0.8,44.8) \\
23.0(1.6,44.4)\end{array}$ & $\begin{array}{c}32] \\
\end{array}$ \\
\hline $\begin{array}{l}\text { Shearer A } \\
(2009)\end{array}$ & - & SureStepFlexx & - & - & $8.7(-18.2,35.6)$ & - & [33] \\
\hline $\begin{array}{l}\text { Ray JG } \\
(2001)\end{array}$ & - & - & One-touch profile & - & - & $0.7(-39.6,41.4)$ & [34] \\
\hline $\begin{array}{l}\text { Denfeld QE } \\
\text { (2011) }\end{array}$ & - & - & Precision Xceedpro & - & - & $12.3(-6.9,31.5)$ & [35] \\
\hline
\end{tabular}

Bias was described as mean difference (95\% confidence interval). ABG, arterial blood gas analyzer; Gluco-C, glucose meters using capillary blood samples, Gluco-

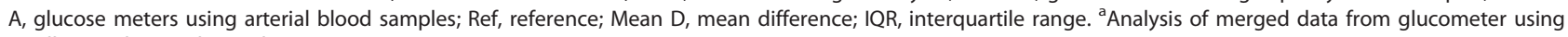
capillary and arterial samples.

a reference were selected for further assessment (Table 1). All of the 11 studies were single-center observational studies. Nine of the 11 studies were prospective studies [15-19,21,23-25], and the other two studies were retrospective studies [20,22]. Totally, 580 patients were included in the 11 studies.

Various types of central laboratory machines were used in the studies. The two methods for blood-glucose 
monitoring are the hexokinase method (Aeroset, Dimention, and Vista device, Au640e and Architect CI 8200) $[15,17,21,22,25]$ and the glucose oxidase method (YSI 2300, Lx-20, Vitros950, and 5.1FS) [16,18-20,23,24]. All machines had traceability to a higher-order reference method.

\section{Accuracy of blood-glucose measurements in the whole glycemic range \\ Arterial blood glucose analyzers}

The accuracy of Glu-ABGs including Cobas B 221 [15], ABL 800 [15], Grem Premiere 3000 [15], ABL715 [16],
RapidLab [17], Chiron 865 [18], Rapidlab 860 [19], and Rapidpoint 405 [20] was assessed in six studies (Table 3). Arterial blood samples were used in all of those studies. The accuracy of Glu-ABGs was assessed by using ISO criteria in one study [17], error-grid analysis in four studies $[15,16,18,20]$, and $20 \%$ error in one study [19].

There were 1,444 assessments in the six studies. The proportion of nonagreement varied from 0 to $42.8 \%$. Five (83.3\%) studies showed good quality of blood-glucose monitoring (nonagreement, $<5 \%$ ). The proportion of nonagreement was $12.5 \%$ in total. Overestimation of blood-glucose concentrations was seen in $12.3 \%$ of all assessments.

Table 3 Agreement of blood-glucose monitoring with each device

\begin{tabular}{|c|c|c|c|c|c|c|c|}
\hline $\begin{array}{l}\text { First author } \\
\text { (year) }\end{array}$ & Devices & $\begin{array}{l}\text { Methods for } \\
\text { assessment }\end{array}$ & $\begin{array}{c}\text { Nonagreement } \\
\text { Proportion } \\
<5 \% \\
\end{array}$ & $\begin{array}{c}\text { Nonagreement } \\
\text { proportion }\end{array}$ & Overestimation & Underestimation & Ref \\
\hline \multicolumn{8}{|l|}{ ABG } \\
\hline $\begin{array}{l}\text { Stadlbauer V } \\
(2010)\end{array}$ & $\begin{array}{c}\text { Cobas B } 221 \\
\text { ABL } 800 \\
\text { Grem Premiere } 3000\end{array}$ & $\begin{array}{l}\text { Clarke } \\
\text { error grid }\end{array}$ & Yes & $\begin{array}{l}0 / 74 \\
(0 \%)\end{array}$ & $\begin{array}{l}0 / 74 \\
(0 \%)\end{array}$ & $\begin{array}{l}0 / 74 \\
(0 \%)\end{array}$ & [15] \\
\hline $\begin{array}{l}\text { Corstjens AM } \\
\text { (2006) }\end{array}$ & ABL715 & $\begin{array}{l}\text { Clarke } \\
\text { error grid }\end{array}$ & No & $\begin{array}{c}178 / 416 \\
(42.8 \%)\end{array}$ & $\begin{array}{l}178 / 416 \\
(42.8 \%)\end{array}$ & $\begin{array}{c}0 / 416 \\
(0 \%)\end{array}$ & {$[16]$} \\
\hline $\begin{array}{l}\text { Hoedemaekers } \\
\text { CW } \\
\text { (2008) }\end{array}$ & RapidLab & ISO & Yes & $\begin{array}{l}0 / 32 \\
(0 \%)\end{array}$ & $\begin{array}{l}0 / 32 \\
(0 \%)\end{array}$ & $\begin{array}{l}0 / 32 \\
(0 \%)\end{array}$ & [17] \\
\hline $\begin{array}{l}\text { Slater-MacLean L } \\
\text { (2008) }\end{array}$ & Chiron865 & $\begin{array}{l}\text { Modified } \\
\text { error grid }\end{array}$ & Yes & $\begin{array}{l}1 / 683 \\
(0.1 \%)\end{array}$ & $\begin{array}{c}0 / 683 \\
(0 \%)\end{array}$ & $\begin{array}{l}1 / 683 \\
(0.1 \%)\end{array}$ & [18] \\
\hline $\begin{array}{l}\text { Kanji S } \\
\text { (2005) }\end{array}$ & RapidLab860 & Within 20\% & Yes & $\begin{array}{l}1 / 115 \\
(0.9 \%)\end{array}$ & $\begin{array}{c}0 / 115 \\
(0 \%)\end{array}$ & $\begin{array}{l}1 / 115 \\
(0.9 \%)\end{array}$ & [19] \\
\hline $\begin{array}{l}\text { Petersen JR } \\
\text { (2008) }\end{array}$ & Rapidpoint405 & $\begin{array}{l}\text { Modified } \\
\text { error grid }\end{array}$ & Yes & $\begin{array}{c}0 / 114 \\
(0 \%)\end{array}$ & $\begin{array}{c}0 / 114 \\
(0 \%)\end{array}$ & $\begin{array}{c}0 / 114 \\
(0 \%)\end{array}$ & [20] \\
\hline Total & & & $\begin{array}{c}5 / 6 \\
(83.3 \%)\end{array}$ & $\begin{array}{c}180 / 1,444 \\
(12.5 \%)\end{array}$ & $\begin{array}{c}178 / 1,444 \\
(12.3 \%)\end{array}$ & $\begin{array}{c}2 / 1,444 \\
(0.1 \%)\end{array}$ & \\
\hline \multicolumn{8}{|l|}{ Gluco-C } \\
\hline $\begin{array}{l}\text { Slater-MacLean L } \\
\text { (2008) }\end{array}$ & $\begin{array}{c}\text { SuperStrepFlexx } \\
\text { Accu-Chek Inform } \\
\text { FreeStyle }\end{array}$ & $\begin{array}{l}\text { Modified } \\
\text { error grid }\end{array}$ & Yes & $\begin{array}{c}24 / 1,656 \\
(1.4 \%)\end{array}$ & $\begin{array}{c}15 / 1,656 \\
(0.9 \%)\end{array}$ & $\begin{array}{l}9 / 1,656 \\
(0.5 \%)\end{array}$ & [18] \\
\hline $\begin{array}{l}\text { Kanji S } \\
(2005)\end{array}$ & Accu-Chek Inform & Within $20 \%$ & No & $\begin{array}{l}32 / 118 \\
(27.1 \%)\end{array}$ & $\begin{array}{l}26 / 118 \\
(22.0 \%)\end{array}$ & $\begin{array}{l}6 / 118 \\
(5.1 \%)\end{array}$ & [19] \\
\hline $\begin{array}{l}\text { Petersen JR } \\
\text { (2008) }\end{array}$ & Accu-Chek Inform & $\begin{array}{l}\text { Modified } \\
\text { error grid }\end{array}$ & No & $\begin{array}{l}23 / 114 \\
(20.2 \%)\end{array}$ & $\begin{array}{l}20 / 114 \\
(17.4 \%)\end{array}$ & $\begin{array}{l}3 / 114 \\
(2.6 \%)\end{array}$ & [20] \\
\hline $\begin{array}{l}\text { Desachy A } \\
\text { (2008) }\end{array}$ & Accu-Chek Sensor & Within $20 \%$ & No & $\begin{array}{l}41 / 273 \\
(15.0 \%) \\
\end{array}$ & $\begin{array}{l}12 / 273 \\
(4.4 \%) \\
\end{array}$ & $\begin{array}{l}29 / 273 \\
(10.6 \%) \\
\end{array}$ & [21] \\
\hline $\begin{array}{l}\text { PulziJúnior SA } \\
\text { (2009) }\end{array}$ & FreeStyle & Within $20 \%$ & No & $\begin{array}{c}9 / 38 \\
(23.4 \%)\end{array}$ & $\begin{array}{c}8 / 38 \\
(21.1 \%)\end{array}$ & $\begin{array}{c}1 / 38 \\
(2.6 \%)\end{array}$ & [22] \\
\hline $\begin{array}{l}\text { Lonjaret L } \\
(2012)\end{array}$ & Contour & Within $20 \%$ & No & $\begin{array}{l}75 / 302 \\
(24.8 \%)\end{array}$ & $\begin{array}{l}8 / 302 \\
(2.6 \%)\end{array}$ & $\begin{array}{l}67 / 302 \\
(22.2 \%)\end{array}$ & [23] \\
\hline $\begin{array}{l}\text { Critchell CD } \\
\text { (2007) }\end{array}$ & Accu-Chek Inform & ISO & No & $\begin{array}{l}53 / 277 \\
(19.1 \%)\end{array}$ & $\begin{array}{l}44 / 277 \\
(15.9 \%)\end{array}$ & $\begin{array}{l}9 / 277 \\
(3.2 \%)\end{array}$ & [24] \\
\hline Total & & & $\begin{array}{c}1 / 7 \\
(14.3 \%)\end{array}$ & $\begin{array}{c}257 / 2,778 \\
(9.3 \%)\end{array}$ & $\begin{array}{c}133 / 2,778 \\
(4.8 \%)\end{array}$ & $\begin{array}{c}124 / 2778 \\
(4.5 \%)\end{array}$ & \\
\hline \multicolumn{8}{|l|}{ Gluco-A } \\
\hline $\begin{array}{l}\text { Slater-MacLean L } \\
\text { (2008) }\end{array}$ & $\begin{array}{l}\text { SuperStrepFlexx } \\
\text { Accu-Chek Inform } \\
\text { FreeStyle }\end{array}$ & $\begin{array}{l}\text { Modified } \\
\text { error grid }\end{array}$ & Yes & $\begin{array}{l}1 / 2,048 \\
(0.05 \%)\end{array}$ & $\begin{array}{c}0 / 2,048 \\
(0 \%)\end{array}$ & $\begin{array}{l}1 / 2,048 \\
(0.05 \%)\end{array}$ & [18] \\
\hline $\begin{array}{l}\text { Kanji S } \\
(2005)\end{array}$ & Accu-Chek Inform & Within $20 \%$ & No & $\begin{array}{l}14 / 113 \\
(12.3 \%)\end{array}$ & $\begin{array}{l}10 / 113 \\
(8.8 \%)\end{array}$ & $\begin{array}{l}4 / 113 \\
(3.5 \%)\end{array}$ & [19] \\
\hline
\end{tabular}


Table 3 Agreement of blood-glucose monitoring with each device (Continued)

\begin{tabular}{|c|c|c|c|c|c|c|c|}
\hline $\begin{array}{l}\text { Petersen JR } \\
\text { (2008) }\end{array}$ & Accu-Chek Inform & $\begin{array}{l}\text { Modified } \\
\text { error grid }\end{array}$ & No & $\begin{array}{l}13 / 114 \\
(11.3 \%)\end{array}$ & $\begin{array}{l}13 / 114 \\
(11.3 \%)\end{array}$ & $\begin{array}{c}0 / 114 \\
(0 \%)\end{array}$ & [20] \\
\hline $\begin{array}{l}\text { Desachy A } \\
\text { (2008) }\end{array}$ & Accu-Chek Sensor & Within 20\% & No & $\begin{array}{l}13 / 232 \\
(5.6 \%)\end{array}$ & n.a. & n.a. & [21] \\
\hline $\begin{array}{l}\text { PulziJúnior SA } \\
\text { (2009) }\end{array}$ & FreeStyle & Within 20\% & No & $\begin{array}{c}3 / 38 \\
(7.9 \%)\end{array}$ & $\begin{array}{l}3 / 38 \\
(7.9 \%)\end{array}$ & $\begin{array}{l}0 / 38 \\
(0)\end{array}$ & [22] \\
\hline $\begin{array}{l}\text { Lonjaret L } \\
\text { (2012) }\end{array}$ & Contour & Within 20\% & No & $\begin{array}{l}35 / 302 \\
(11.6 \%)\end{array}$ & $\begin{array}{l}7 / 302 \\
(2.3 \%)\end{array}$ & $\begin{array}{c}28 / 302 \\
(9.3 \%) \\
\end{array}$ & [23] \\
\hline $\begin{array}{l}\text { Meynaar IA } \\
(2009)\end{array}$ & Accu-Check Inform & Within $20 \%$ & No & $\begin{array}{c}22 / 239 \\
(9.6 \%)\end{array}$ & $\begin{array}{l}3 / 239 \\
(1.3 \%)\end{array}$ & $\begin{array}{c}19 / 239 \\
(7.9 \%)\end{array}$ & [25] \\
\hline Total & & & $\begin{array}{c}1 / 7 \\
(14.3 \%)\end{array}$ & $\begin{array}{c}101 / 3,086 \\
(3.3 \%)\end{array}$ & $\begin{array}{c}36 / 2,854 \\
(1.3 \%)\end{array}$ & $\begin{array}{c}52 / 2,854 \\
(1.8 \%)\end{array}$ & \\
\hline
\end{tabular}

ABG, arterial blood gas analyzer; Gluco-C, glucose meters using capillary blood samples; Gluco-A, glucose meters using arterial blood samples; Ref, reference.

\section{Glucose meters using capillary blood samples}

In seven studies, the accuracy of Gluco-C, including SuperStrepFlexx [18], AccuCheck Inform [18-20,24], FreeStyle [18,22], Accu-Chek Sensor [21], and Contour [23], was assessed (Table 3). The accuracy of Gluco-C was assessed by using ISO criteria in one study [24], error-grid analysis in two studies [18,20], and $20 \%$ error in four studies [19,21-23].

In the 2,778 assessments in the seven studies, the proportion of nonagreement varied from $1.4 \%$ to $27.1 \%$. One study (14.3\%) showed a good quality of blood-glucose monitoring [18]. The proportion of nonagreement was 9.3\%. Overestimation of blood-glucose concentrations was seen in $4.8 \%$ of all assessments.

\section{Glucose meters using arterial blood samples}

In seven studies, the accuracy of Gluco-A, including SuperStrepFlexx [18], AccuCheck Inform [18-20,25], FreeStyle [18,22], Accu-Chek Sensor [21], and Contour [23] was assessed (Table 3). The accuracy of Gluco-A was assessed by using error-grid analysis in two studies $[18,20]$ and $20 \%$ error in five studies $[19,21-23,25]$.

In the seven studies, 3,086 assessments were done. The proportion of nonagreement varied from 0 to $12.3 \%$. One study (14.3\%) showed good quality of blood-glucose monitoring [18]. The proportion of nonagreement was $3.3 \%(n=101)$. Overestimation of blood-glucose values was seen in $1.3 \%$ of all assessments.

\section{Meta-analysis to compare the accuracy of devices}

In three studies, the accuracy of ABGs and that of glucose meters were compared simultaneously [18-20]. Glu-ABGs were significantly more accurate than Gluco-C (odds ratio for nonagreement, $0.04 ; P<0.001$ ) (Figure 2A). Glu-ABGs tended to be more accurate, but not significantly more accurate, than Gluco-A (odds ratio for nonagreement, $0.17 ; P=0.20$ ) (Figure $2 \mathrm{~B}$ ).

In six studies, the accuracy of Gluco-A and that of Gluco-C [18-23] were compared. Gluco-A results were significantly more accurate than those of Gluco-C (odds ratio for nonagreement, $0.36 ; P<0.001$ ) (Figure $2 \mathrm{C}$ ).
Accuracy of blood-glucose measurements in the hypoglycemic range

The accuracy of point of blood-glucose monitoring in the hypoglycemic range was assessed for Glu-ABGs in two studies [16,19], for Gluco-C in three studies $[19,23,24]$, and for Gluco-A in three studies [19,23,25] (Table 4). The total number of assessments was 157 (59 assessments for ABGs, 52 assessments for Gluco-C, and 46 assessments for Gluco-A).

For ABGs, 13 of the 59 blood-glucose measurements were outside the agreement range $(22.0 \%)$, and all of them overestimated blood-glucose values $(22.0 \%)$. One study by Kanji et al. [19] showed a high level of accuracy of ABGs in the hypoglycemic range (nonagreement, none of 37) [19]. For Gluco-C, 26 of the 77 blood-glucose measurements were outside the agreement range (33.8\%). Overestimation of blood-glucose values was seen in 15 measurements (19.5\%). For Gluco-A, 14 of the 71 bloodglucose measurements were outside the agreement range (19.7\%). Overestimation of blood-glucose values was seen in eight (11.3\%) measurements.

Blood-glucose measurements in the hypoglycemic range were less accurate than were those in the nonhypoglycemic range among all three devices (odds ratio for error, Glu-ABGs, 1.86, $P=0.15$; Gluco-C, $1.84, P=0.03$; Gluco-A, 2.33, $P=0.02$ ).

\section{Factors associated with error of blood-glucose measurements}

In six studies, risk factors for inaccuracy of glucose measurements were determined (five for Gluco- $C$, five for Gluco-A, and none for ABGs) [20-25] (Table 5). Patient's factors (sex, body mass index, severity of illness, and presence of sepsis and/or diabetes), except for age, were not significantly related to inaccuracy. Young age was significantly associated with increased risk of nonagreement for Gluco-C in one study [23]. No laboratory data (albumin, lactate, $\mathrm{PaCO}_{2}, \mathrm{PaO}_{2}, \mathrm{pH}$, and hematocrit) were associated with inaccuracy. 


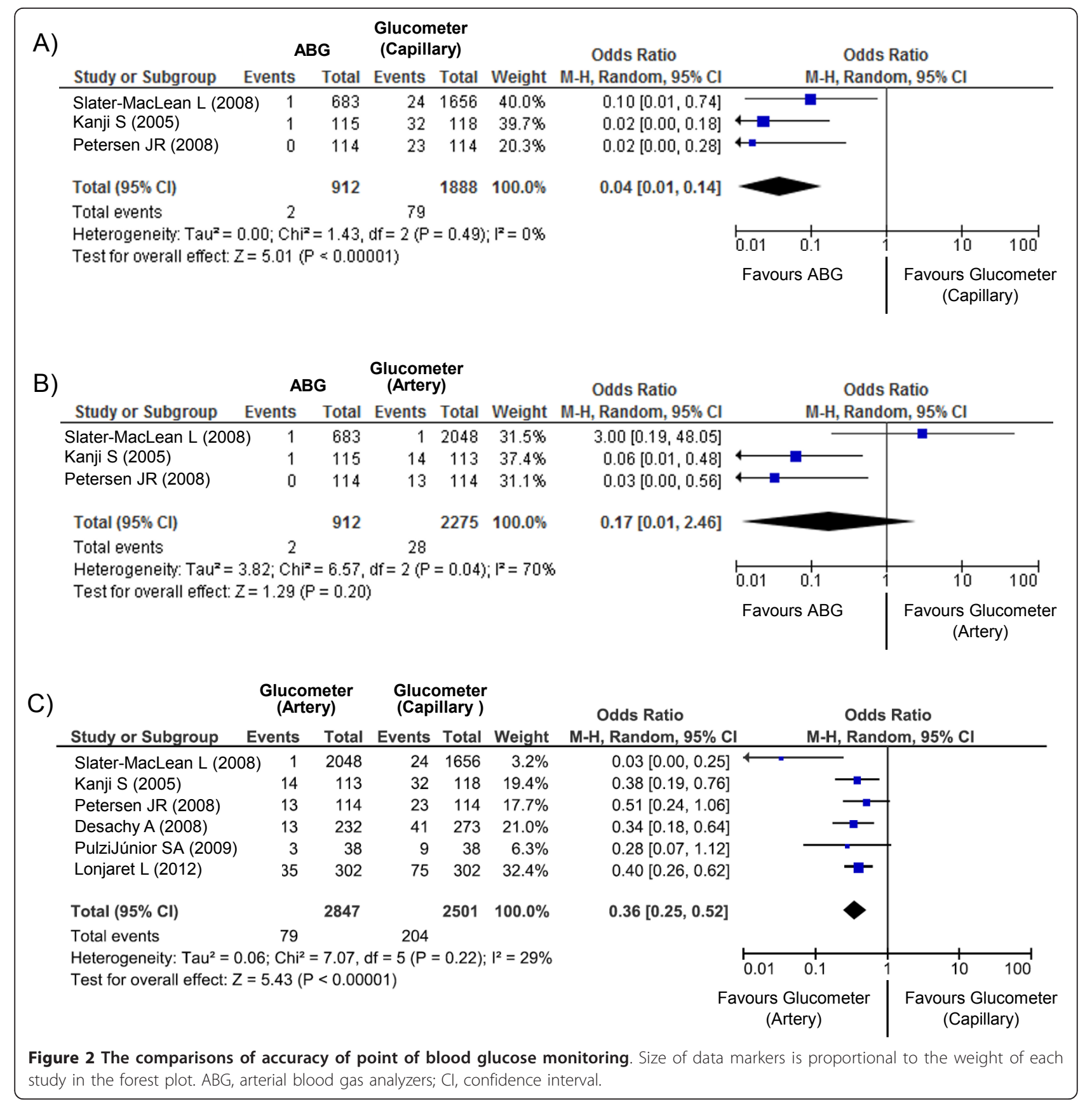

Table 4 Agreement of each method in the hypoglycemic range

\begin{tabular}{|c|c|c|c|c|c|c|c|c|c|c|c|}
\hline $\begin{array}{l}\text { First author } \\
\text { (year) }\end{array}$ & Devices & Methods & $\begin{array}{l}\text { Definition } \\
\text { (mg/dl) }\end{array}$ & $\begin{array}{c}\text { No of } \\
\text { samples }\end{array}$ & $\begin{array}{l}\text { Non } \\
\text { Agreement } \\
\text { proportion } \\
<5 \%\end{array}$ & $\begin{array}{c}\text { Non } \\
\text { agreement } \\
\text { proportion }\end{array}$ & $\begin{array}{c}\text { Over } \\
\text { estimation }\end{array}$ & $\begin{array}{l}\text { Under } \\
\text { estimation }\end{array}$ & $\begin{array}{c}\text { Non } \\
\text { agreement } \\
\text { in } \\
\text { non-hypo } \\
\text { range }\end{array}$ & $\begin{array}{l}\text { Odds ratio } \\
(95 \% \mathrm{Cl})\end{array}$ & Ref \\
\hline \multicolumn{12}{|l|}{ ABG } \\
\hline $\begin{array}{l}\text { Corstjens AM } \\
(2006)\end{array}$ & ABL 715 & $\begin{array}{c}\text { Zone } \\
\text { A }\end{array}$ & 70 & 22 & No & $\begin{array}{l}13 / 22 \\
(59 \%) \\
\end{array}$ & $\begin{array}{l}13 / 22 \\
(59 \%) \\
\end{array}$ & $\begin{array}{c}0 / 22 \\
(0) \\
\end{array}$ & $\begin{array}{c}165 / 394 \\
(41.9 \%) \\
\end{array}$ & $\begin{array}{c}2.00 \\
(0.84,4.80) \\
\end{array}$ & [16] \\
\hline $\begin{array}{l}\text { Kanji S } \\
(2005)\end{array}$ & RapidLab 860 & $\begin{array}{l}\text { Within } \\
20 \%\end{array}$ & 81 & 37 & Yes & $\begin{array}{l}0 / 37 \\
(0)\end{array}$ & $\begin{array}{l}0 / 37 \\
(0)\end{array}$ & $\begin{array}{l}0 / 37 \\
(0)\end{array}$ & $\begin{array}{c}1 / 78 \\
(1.3 \%)\end{array}$ & $\begin{array}{c}0.69 \\
(0.03,17.3)\end{array}$ & [19] \\
\hline
\end{tabular}


Table 4 Agreement of each method in the hypoglycemic range (Continued)

\begin{tabular}{|c|c|c|c|c|c|c|c|c|c|c|c|}
\hline Total & & & & 59 & $\begin{array}{c}1 / 2 \\
(50 \%)\end{array}$ & $\begin{array}{c}13 / 59 \\
(22.0 \%)\end{array}$ & $\begin{array}{c}13 / 59 \\
(22.0 \%)\end{array}$ & $\begin{array}{c}0 / 59 \\
(0)\end{array}$ & $\begin{array}{c}166 / 472 \\
(35.2 \%)\end{array}$ & $\begin{array}{c}1.86 \\
(0.80,4.33)\end{array}$ & \\
\hline \multicolumn{12}{|l|}{ Gluco-C } \\
\hline $\begin{array}{l}\text { Kanji S } \\
(2005) \\
\end{array}$ & $\begin{array}{l}\text { Accu-Chek } \\
\text { Inform }\end{array}$ & $\begin{array}{l}\text { Within } \\
20 \%\end{array}$ & 81 & 38 & No & $\begin{array}{l}14 / 38 \\
(38 \%)\end{array}$ & $\begin{array}{l}11 / 38 \\
(30 \%)\end{array}$ & $\begin{array}{l}3 / 38 \\
(8 \%) \\
\end{array}$ & $\begin{array}{c}18 / 80 \\
(22.5 \%) \\
\end{array}$ & $\begin{array}{c}2.30 \\
(1.00,5.32) \\
\end{array}$ & [19] \\
\hline $\begin{array}{l}\text { Lonjaret L } \\
(2012)\end{array}$ & Contour & $\begin{array}{l}\text { Within } \\
20 \%\end{array}$ & 81 & 25 & No & $\begin{array}{l}8 / 25 \\
(32 \%)\end{array}$ & $\begin{array}{l}3 / 25 \\
(12 \%)\end{array}$ & $\begin{array}{l}5 / 25 \\
(20 \%)\end{array}$ & $\begin{array}{l}67 / 277 \\
(24.2 \%)\end{array}$ & $\begin{array}{c}1.47 \\
(0.61,3.57)\end{array}$ & [23] \\
\hline $\begin{array}{l}\text { Critchell CD } \\
(2007)\end{array}$ & $\begin{array}{l}\text { Accu-Chek } \\
\text { Inform }\end{array}$ & $\begin{array}{c}<15 \\
\mathrm{mg} / \mathrm{dl}\end{array}$ & 75 & 14 & No & $\begin{array}{c}4 / 14 \\
(29 \%)\end{array}$ & $\begin{array}{l}1 / 14 \\
(7 \%)\end{array}$ & $\begin{array}{c}3 / 14 \\
(21 \%) \\
\end{array}$ & $\begin{array}{l}49 / 263 \\
(18.6 \%) \\
\end{array}$ & $\begin{array}{c}1.75 \\
(0.53,5.80)\end{array}$ & [24] \\
\hline Total & & & & 77 & $\begin{array}{c}0 / 3 \\
(0 \%) \\
\end{array}$ & $\begin{array}{c}26 / 77 \\
(33.8 \%)\end{array}$ & $\begin{array}{c}15 / 77 \\
(19.5 \%) \\
\end{array}$ & $\begin{array}{c}11 / 77 \\
(14.3 \%) \\
\end{array}$ & $\begin{array}{l}134 / 620 \\
(21.6 \%)\end{array}$ & $\begin{array}{c}1.84 \\
(1.07,3.16) \\
\end{array}$ & \\
\hline \multicolumn{12}{|l|}{ Gluco-A } \\
\hline $\begin{array}{l}\text { Kanji S } \\
(2005)\end{array}$ & $\begin{array}{l}\text { Accu-Chek } \\
\text { Inform }\end{array}$ & $\begin{array}{l}\text { Within } \\
20 \%\end{array}$ & 81 & 36 & No & $\begin{array}{c}8 / 36 \\
(22 \%)\end{array}$ & $\begin{array}{c}6 / 36 \\
(17 \%)\end{array}$ & $\begin{array}{l}2 / 36 \\
(6 \%)\end{array}$ & $\begin{array}{c}6 / 77 \\
(7.8 \%)\end{array}$ & $\begin{array}{c}3.38 \\
(1.08,10.6)\end{array}$ & [19] \\
\hline $\begin{array}{l}\text { Lonjaret L } \\
(2012)\end{array}$ & Contour & $\begin{array}{l}\text { Within } \\
20 \%\end{array}$ & 81 & 25 & No & $\begin{array}{l}5 / 25 \\
(20 \%)\end{array}$ & $\begin{array}{l}1 / 25 \\
(4 \%)\end{array}$ & $\begin{array}{c}4 / 25 \\
(16 \%)\end{array}$ & $\begin{array}{l}30 / 277 \\
(10.8 \%)\end{array}$ & $\begin{array}{c}2.06 \\
(0.72,5.89)\end{array}$ & [23] \\
\hline $\begin{array}{l}\text { Meynaar IA } \\
(2009)\end{array}$ & $\begin{array}{l}\text { Accu-Check } \\
\text { Inform }\end{array}$ & $\begin{array}{c}<15 \\
\mathrm{mg} / \mathrm{dl}\end{array}$ & 75 & 10 & No & $\begin{array}{c}1 / 10 \\
(10 \%)\end{array}$ & $\begin{array}{c}1 / 10 \\
(10 \%)\end{array}$ & $\begin{array}{l}0 / 10 \\
(0 \%)\end{array}$ & $\begin{array}{c}21 / 229 \\
(9.2 \%)\end{array}$ & $\begin{array}{c}1.10 \\
(0.13,9.12)\end{array}$ & [25] \\
\hline Total & & & & 71 & $\begin{array}{c}0 / 3 \\
(0 \%)\end{array}$ & $\begin{array}{c}14 / 71 \\
(19.7 \%)\end{array}$ & $\begin{array}{c}8 / 71 \\
(11.3 \%)\end{array}$ & $\begin{array}{c}6 / 71 \\
(8.4 \%)\end{array}$ & $\begin{array}{c}57 / 583 \\
(9.8 \%)\end{array}$ & $\begin{array}{c}2.33 \\
(1.13,4.83)\end{array}$ & \\
\hline
\end{tabular}

Definition, Definition of hypoglycemia; No of samples, Number of samples in hypoglycemic range; non-hypo, non-hypoglycemic. ABG, arterial blood gas analyzer; Gluco-C, glucose meters using capillary blood samples; Gluco-A, glucose meters using arterial blood samples; $\mathrm{Cl}$, confidence interval; Ref, reference.

For Gluco-C, low perfusion index [36], use of a vasopressor $[22,24]$ and presence of edema $[20,24]$ were significantly associated with inaccuracy. For Gluco-A, use of a vasopressor [23], low peripheral perfusion, and low mean arterial pressure [21] were associated with inaccuracy.

\section{Studies in which agreement of criteria other than "within $20 \%$ " was assessed}

Our literature review retrieved three studies in which agreement of criteria other than "within 20\%" was assessed: one study used within $20 \mathrm{mg} / \mathrm{dl}$ from the reference [26], and two studies used within $10 \%$ of reference methods for evaluation $[27,28]$ (Table 6). No study showed a good quality of blood-glucose monitoring. One study $(n=20)$ showed that blood-glucose measurements by Accu-Chek Inform using arterial blood samples were less accurate than those using capillary blood samples (odds ratio for incidence of nonagreement, 2.21; $P=0.02$ ) [27]. Another study showed that accuracy of measurements with glucose meters by using arterial blood samples were significantly varied among devices (incidence of nonagreement (StatStrip = reference): Accu-Chek Inform: odds ratio, 5.2; $P<0.001$, Precision PCx: odds ratio, 15.2; $P<0.001$; SureStepFlexx, odds ratio, $4.3 ; P<0.001)$ [28].

\section{Discussion}

Although several reviews focused on the accuracy of point of blood-glucose monitoring in critically ill patients $[10,37,38]$, our review is the first systematic review for this issue. Our review shows comparisons among devices and between hypo- and non-hypoglycemic ranges, as well as problems in studies including variation of references and insufficient data for a hypoglycemic range.

Although available data are often heterogeneous and insufficient for meta-analysis, we found that the accuracy of blood-glucose monitoring might vary, especially according to the device, site of blood sampling, and glucose range. With our systematic analysis of the 11 retrieved articles, we considered that, despite the limitation of data, some statements can be made to help establish current knowledge of the accuracy of point of blood-glucose monitoring in critically ill adult patients.

\section{Statement 1: Type of central laboratory machine (reference) is highly variable}

The type of central laboratory machine varied among the studies. Although all central machines used in the 11 studies have traceability of blood-glucose monitoring, it is unclear whether these machines are equally accurate. Thus, it is difficult to interpret whether the type of laboratory machine influenced the accuracy of point of bloodglucose monitoring. If the central laboratory machine does not have metrologic traceability for blood-glucose monitoring, it should be the case for quality-insurance programs requirements.

Statement 2: In few studies was the accuracy of ABGs compared with that of a glucose meter simultaneously In the variation of reference as in statement 1 , the study to compare the accuracy among Glu-ABGs, Gluco-C, 
Table $\mathbf{5}$ Risk factors for inaccuracy of glucose monitoring

\begin{tabular}{|c|c|c|c|c|c|c|c|c|c|c|c|c|c|c|c|c|c|c|c|c|c|}
\hline First author (year) & Sex & Age & BMI & $\begin{array}{c}\text { Severity } \\
\text { of } \\
\text { illness }\end{array}$ & Sepsis & DM & Alb & Lac & $\mathrm{PaO}_{2}$ & $\mathrm{PaCO}_{2}$ & $\mathrm{pH}$ & $\mathrm{Ht}$ & $\begin{array}{c}\text { Use } \\
\text { of } \\
\text { insulin }\end{array}$ & $\begin{array}{l}\text { Use } \\
\text { of } \\
\text { steroid }\end{array}$ & P.I. & HR & $\begin{array}{c}\text { Use } \\
\text { of } \\
\text { Vaso- } \\
\text { pressor }\end{array}$ & $\begin{array}{l}\text { Low } \\
\text { peripheral } \\
\text { perfusion }\end{array}$ & $\begin{array}{l}\text { Low } \\
\text { MAP }\end{array}$ & Edema & Ref \\
\hline
\end{tabular}

\begin{tabular}{|c|c|c|c|c|c|c|c|c|c|c|c|c|c|c|c|c|c|c|c|c|c|}
\hline \multicolumn{22}{|l|}{ Gluco-C } \\
\hline $\begin{array}{l}\text { Petersen JR } \\
\text { (2008) }\end{array}$ & - & - & - & - & - & - & - & - & - & - & - & - & - & - & - & - & - & - & - & + & [20] \\
\hline $\begin{array}{l}\text { Desachy A } \\
\text { (2008) }\end{array}$ & - & - & - & $\circ$ & - & $\circ$ & - & - & - & - & - & ० & - & - & + & ० & - & $\circ$ & $\circ$ & - & [21] \\
\hline $\begin{array}{l}\text { PulziJúnior SA } \\
\text { (2009) }\end{array}$ & - & - & - & - & - & - & - & - & - & - & - & - & - & - & - & - & + & $\circ$ & - & - & [22] \\
\hline $\begin{array}{l}\text { Critchell CD } \\
\text { (2007) }\end{array}$ & o & o & - & 0 & 0 & - & o & - & 0 & 0 & o & o & - & 0 & - & - & + & - & - & + & [24] \\
\hline $\begin{array}{l}\text { Lonjaret L } \\
\text { (2012) }\end{array}$ & $\circ$ & + & 0 & $\circ$ & - & - & - & o & - & - & o & - & + & - & - & - & o & - & - & - & [23] \\
\hline \multicolumn{22}{|l|}{ Gluco-A } \\
\hline $\begin{array}{l}\text { Petersen JR } \\
\text { (2008) }\end{array}$ & - & - & - & - & - & - & - & - & - & - & - & - & - & - & - & - & - & - & - & 0 & [20] \\
\hline $\begin{array}{l}\text { Desachy A } \\
\text { (2008) }\end{array}$ & - & - & - & o & - & o & - & - & - & - & - & o & - & - & ○ & ० & - & + & + & - & [21] \\
\hline $\begin{array}{l}\text { PulziJúnior SA } \\
\text { (2009) }\end{array}$ & - & - & - & - & - & - & - & - & - & - & - & - & - & - & - & - & o & $\circ$ & - & - & [22] \\
\hline $\begin{array}{l}\text { Meynaar IA } \\
\text { (2009) }\end{array}$ & - & - & - & - & - & - & - & - & - & - & - & o & - & - & - & - & - & - & - & - & [25] \\
\hline $\begin{array}{l}\text { Lonjaret L } \\
\text { (2012) }\end{array}$ & $\circ$ & o & o & $\circ$ & - & - & - & o & - & - & o & - & + & - & - & - & + & - & - & - & [23] \\
\hline
\end{tabular}

BMI, Body mass index; DM, diabetes mellitus, Alb, serum albumin concentration; Lac, lactate concentration; Ht, hematocrit; P.l, perfusion index, MAP, mean arterial pressure, Ref, reference; Gluco-C, glucose meter; using capillary blood samples; Gluco-A, glucose meters using arterial blood samples. +, factor significantly associated with disagreement of blood glucose monitoring. O, factor not significantly associated with

disagreement of blood glucose monitoring. 
Table 6 Three studies in which agreement of criteria other than "within 20\%" was assessed

\begin{tabular}{|c|c|c|c|c|c|c|}
\hline & First author (year) & Devices & Methods for assessment & $\begin{array}{l}\text { Nonagreement } \\
\text { proportion } \\
<5 \%\end{array}$ & $\begin{array}{l}\text { Nonagreement } \\
\text { proportion }\end{array}$ & Ref \\
\hline \multirow[t]{2}{*}{ Gluco-C } & $\begin{array}{l}\text { Cook A } \\
(2009)\end{array}$ & SuperStepFexx & Within $20 \mathrm{mg} / \mathrm{dl}$ & No & 10/64 (15.6\%) & {$[26]$} \\
\hline & $\begin{array}{l}\text { Karon BS } \\
(2007)\end{array}$ & Accu-Chek inform & Within 10\% & No & 25/96 (26.0\%) & {$[27]$} \\
\hline \multirow[t]{2}{*}{ Gluco-A } & $\begin{array}{l}\text { Karon BS } \\
(2007)\end{array}$ & Accu-Chek inform & Within $10 \%$ & No & 42/96 (43.8\%) & {$[27]$} \\
\hline & $\begin{array}{l}\text { Karon } \\
(2008)\end{array}$ & $\begin{array}{l}\text { Accu-Chek Infor } \\
\text { Precision PCx } \\
\text { SureStepFlexx } \\
\text { StatStrip }\end{array}$ & Within 10\% & No & $\begin{array}{l}58 / 185(31.4 \%) \\
106 / 185(57.3 \%) \\
51 / 185(27.6 \%) \\
15 / 185(8.1 \%)\end{array}$ & [28] \\
\hline
\end{tabular}

and Gluco-A is essentially relevant. However, in only three studies were the accuracies of these three compared [18-20].

\section{Statement 3: Accuracy of ABG analyzers might vary among devices}

The proportion of nonagreement in Glu-ABGs varied widely (0 to $42.8 \%$ ). Although five of the six studies showed good quality of Glu-ABGs, and the range of limits of agreements for Glu-ABGs (minimum of $19 \mathrm{mg} / \mathrm{dl}$, maximum of $39 \mathrm{mg} / \mathrm{dl}$ ) was smaller than those for Gluco-C and Gluco-A, one study showed overestimation by Glu-ABGs in $42.8 \%$ of the samples. Although it is unclear whether the type of central laboratory machine, conditions of the measurement, or other unknown mechanisms affected the results of that study, the results suggested that accuracy of Glu-ABGs might vary among devices. Thus, it is recommended that each institution confirm the accuracy of their ABGs for blood-glucose monitoring.

Statement 4: ABGs and a glucose meter using arterial blood were significantly more accurate than a glucose meter using capillary blood

Glu-ABGs and Gluco-A were significantly more accurate than Gluco-C. Even when we included studies using criteria other than within $20 \%$, the finding did not change (odds ratio for nonagreement, $0.43 ; P=0.01$ ). Thus, for blood-glucose measurements in critically ill adult patients, arterial blood samples should be used rather than capillary blood samples.

Statement 5: Blood-glucose monitoring with ABG analyzers tends to be more accurate than that with glucose meters using arterial blood

Our meta-analysis showed that Glu-ABGs tend to be more accurate than Gluco-A $(P=0.20)$. Additionally, the range of limits of agreements in Glu-ABG was smaller than that in Gluco-A. These results suggest that Glu-ABGs might be more appropriate than Gluco-A.

However, it should be noted that the accuracy of Gluco-A varied among studies, as stated earlier, and in only three studies were they compared, and the results were conflicting (odds ratios for error, 0.03 to 3.00). Thus, further studies are needed to determine whether Glu-ABGs, Gluco-A, or both can be recommended for blood-glucose monitoring in a critically ill setting.

Statement 6: Information on the accuracy of bloodglucose measurement in the hypoglycemia range is not sufficient

Although more than 6,000 samples were assessed for the accuracy of blood-glucose measurements (ABG, 1,360; Glu-C, 2,858; Glu-A, 3,086), about 70 samples were in the hypoglycemic range in each method (ABG, 58; GluC, 77; Glu-A, 81). This number of samples is not sufficient to compare between devices and determine the risk factors of error. Therefore, further studies are needed for blood-glucose measurements in the hypoglycemic range.

Statement 7: Blood-glucose monitoring in the hypoglycemic range is less accurate than that in the nonhypoglycemic range

Because many studies have shown that even mild hypoglycemia is significantly associated with increase in mortality $[39,40]$, accuracy of blood-glucose monitoring in the hypoglycemic range is important. Although little information is available for the hypoglycemic range, as stated earlier, our results showed that the incidences of errors in the hypoglycemic range were higher than those in the nonhypoglycemic ranges.

Regardless of the method used for blood-glucose monitoring, we should be aware that a greater possibility of errors exists in the hypoglycemic range than in the nonhypoglycemic range. We should confirm blood glucose 
concentrations by using Glu-lab when we obtain bloodglucose values within or near the hypoglycemic range.

\section{Statement 8: Unstable hemodynamics and insulin infusion might increase the risk of errors in blood-glucose monitoring by using a glucose meter}

Unstable hemodynamics (low perfusion index, use of a vasopressor, presence of edema, and low mean arterial pressure) and insulin infusion were associated with increased risk of inaccuracy. These factors might decrease peripheral blood-glucose concentrations through microcirculatory disturbance and increased tissue glucose consumption $[41,42]$. Therefore, physicians should avoid using either Gluco-A and Gluco-C in patients with unstable hemodynamics and/or receiving insulin infusion.

\section{Limitations}

Our systematic review has some limitations. Our literature search was performed by using only MEDLINE and PubMed and was performed by only one author. The use of other important databases, such as the Cochrane systematic reviews database, and selection by multiple authors might have made the literature review more comprehensive. We also excluded non-English-language reports, abstracts, and unpublished studies. Thus, some findings may have been missed. However, the selection was done with preset inclusion criteria and a careful search of bibliographies so as to minimize selection bias.

\section{Conclusions}

Our literature review showed that ABGs were significantly more accurate than glucose meters using capillary blood and tended to be more accurate than glucose meters using arterial blood. However, these results should be interpreted with caution because of the large variation of accuracy among devices. Because blood-glucose monitoring was less accurate within or near the hypoglycemic range, especially in patients with unstable hemodynamics or receiving insulin infusion, we should aware that current blood-glucose monitoring technology has not reached a high enough degree of accuracy and reliability to lead to appropriate glucose control in critically ill patients.

\section{Key messages}

- Accuracy of blood-glucose measurements using arterial blood gas analyzers might vary among devices.

- Blood-glucose monitoring with ABG analyzers tends to be more accurate than that by glucose meters with arterial blood.

- Arterial blood samples should be used rather than capillary blood sample for blood-glucose measurements in adult critically ill patients.
- In the hypoglycemic range, blood-glucose monitoring is more inaccurate than that in the nonhypoglycemic range.

- Unstable hemodynamics and insulin infusion might increase the risk of error in blood-glucose monitoring with a glucose meter.

\section{Abbreviations}

ABGs: arterial blood gas analyzers; Glu-ABGs: blood-glucose measurements by $A B G s ;$ Gluco-A: blood-glucose measurements with glucose meters by using arterial blood samples; Gluco-C: blood-glucose measurements with glucose meters by using capillary blood samples; Glu-lab: central laboratory blood glucose measurements by using plasma; ISO: International Organization for Standardization.

\section{Authors' contributions}

ME and MK conceived the study. ME, JK, and MK participated in the design of the study. SI performed systematic literature search. SI and ME retrieved relevant information from selected articles. SI and ME performed the statistical analyses. SI, ME, JK, and KM participated in data interpretation and drafted the manuscript. All authors read and approved the final manuscript.

\section{Competing interests}

The authors declare that they have no competing interests.

\section{Author details}

${ }^{1}$ Department of Emergency and Critical Care Medicine, Tokai University, School of Medicine, 143 Shimokasuya, Isehara-shi, Kanagawa, 259-1193, Japan. ${ }^{2}$ Department of Anesthesiology and Resuscitology, Okayama University Hospital, 2-5-1 Shikatachou, Okayama, Okayama 700-8525, Japan. ${ }^{3}$ Department of Emergency and Critical Care Medicine, Hyogo College of Medicine, 1-1, Mukogawa-cho, Nishinomiya, Hyogo, 663-8501 Japan.

Received: 29 October 2012 Revised: 25 February 2013

Accepted: 12 March 2013 Published: 18 March 2013

\section{References}

1. Egi M, Finfer S, Bellomo R: Glycemic control in the ICU. Chest 2011 140:212-220

2. NICE-SUGAR Study Investigators, Finfer $S$, Chittock DR, Su SY, Blair D, Foster D, Dhingra V, Bellomo R, Cook D, Dodek P, Henderson WR, Hébert PC, Heritier S, Heyland DK, McArthur C, McDonald E, Mitchell I, Myburgh JA, Norton R, Potter J, Robinson BG, Ronco JJ: Intensive versus conventional glucose control in critically ill patients. N Engl J Med 2009, 360:1283-1297.

3. Friedrich JO, Chant C, Adhikari NK: Does intensive insulin therapy really reduce mortality in critically ill surgical patients? A reanalysis of metaanalytic data. Crit Care 2010, 14:324.

4. Griesdale DE, de Souza RJ, van Dam RM, Heyland DK, Cook DJ, Malhotra A, Dhaliwal R, Henderson WR, Chittock DR, Finfer S, Talmor D: Intensive insulin therapy and mortality among critically ill patients: a metaanalysis including NICE-SUGAR study data. CMAJ 2009, 180:821-827.

5. Marik PE, Preiser JC: Toward understanding tight glycemic control in the ICU: a systematic review and metaanalysis. Chest 2010, 137:544-551.

6. Moghissi ES: Reexamining the evidence for inpatient glucose control: new recommendations for glycemic targets. Am J Health Syst Pharm 2010, 67:S3-8.

7. American Diabetes Association: Standards of medical care in diabetes: 2010. Diabetes Care 33:S11-S61.

8. Neely RD, Kiwanuka JB, Hadden DR: Influence of sample type on the interpretation of the oral glucose tolerance test for gestational diabetes mellitus. Diabetic Med 1991, 8:129-134.

9. D'Orazio P, Burnett RW, Fogh-Andersen N, Jacobs E, Kuwa K, Külpmann WR, Larsson L, Lewenstam A, Maas AH, Mager G, Naskalski JW, Okorodudu AO, International Federation of Clinical Chemistry Scientific Division Working Group on Selective Electrodes and Point of Care Testing: Approved IFCC recommendation on reporting results for blood glucose (abbreviated). Clin Chem 2005, 51:1573-1576. 
10. Pitkin $A D$, Rice $M J$ : Challenges to glycemic measurement in the perioperative and critically ill patient: a review. J Diabetes Sci Technol 2009, 3:1270-1281.

11. Scott MG, Bruns DE, Boyd JC, Sacks DB: Tight glucose control in the intensive care unit: are glucose meters up to the task? Clin Chem 2009, 55:18-20.

12. Stroup DF, Berlin JA, Morton SC, Olkin I, Williamson GD, Rennie D, Moher D, Becker BJ, Sipe TA, Thacker SB: Meta-analysis of observational studies in epidemiology: a proposal for reporting: Meta-analysis Of Observational Studies in Epidemiology (MOOSE) group. JAMA 2000, 283:2008-2012.

13. Higgins JP, Thompson SG, Deeks JJ, Altman DG: Measuring inconsistency in meta-analyses. BMJ 2003, 327:557-560.

14. Sutton AJ, Higgins JP: Recent developments in meta-analysis. Statistics Med 2008, 27:625-650.

15. Stadlbauer V, Wallner S, Stojakovic T, Smolle KH: Comparison of 3 different multianalyte point-of-care devices during clinical routine on a medical intensive care unit. J Crit Care 2011, 26:433, e431-411.

16. Corstjens AM, Ligtenberg JJ, van der Horst IC, Spanjersberg R, Lind JS, Tulleken JE, Meertens JH, Zijlstra JG: Accuracy and feasibility of point-ofcare and continuous blood glucose analysis in critically ill ICU patients. Crit Care 2006, 10:R135.

17. Hoedemaekers CW, Klein Gunnewiek JM, Prinsen MA, Willems $J$, Van der Hoeven JG: Accuracy of bedside glucose measurement from three glucometers in critically ill patients. Crit Care Med 2008, 36:3062-3066.

18. Slater-MacLean L, Cembrowski G, Chin D, Shalapay C, Binette T, Hegadoren K, Newburn-Cook C: Accuracy of glycemic measurements in the critically ill. Diabetes Technol Ther 2008, 10:169-177.

19. Kanji S, Buffie J, Hutton B, Bunting PS, Singh A, McDonald K, Fergusson D, McIntyre LA, Hebert PC: Reliability of point-of-care testing for glucose measurement in critically ill adults. Crit Care Med 2005, 33:2778-2785.

20. Petersen JR, Graves DF, Tacker DH, Okorodudu AO, Mohammad AA, Cardenas VJ Jr: Comparison of POCT and central laboratory blood glucose results using arterial, capillary, and venous samples from MICU patients on a tight glycemic protocol. Clin Chim Acta 2008, 396:10-13.

21. Desachy A, Vuagnat AC, Ghazali AD, Baudin OT, Longuet OH, Calvat SN, Gissot V: Accuracy of bedside glucometry in critically ill patients: influence of clinical characteristics and perfusion index. Mayo Clin Proc 2008, 83:400-405.

22. Pulzi Junior SA, Assuncao MS, Mazza BF, Fernandes Hda S, Jackiu M Freitas FG, Machado FR: Accuracy of different methods for blood glucose measurement in critically ill patients. Sao Paulo Med J 2009, 127:259-265.

23. Lonjaret L, Claverie V, Berard E, Riu-Poulenc B, Geeraerts T, Genestal M, Fourcade O: Relative accuracy of arterial and capillary glucose meter measurements in critically ill patients. Diabetes Metab 2012, 38:230-235.

24. Critchell CD, Savarese V, Callahan A, Aboud C, Jabbour S, Marik P: Accuracy of bedside capillary blood glucose measurements in critically ill patients. Intensive Care Med 2007, 33:2079-2084.

25. Meynaar IA, van Spreuwel M, Tangkau PL, Dawson L, Sleeswijk Visser S, Rijks L, Vlieland TV: Accuracy of AccuChek glucose measurement in intensive care patients. Crit Care Med 2009, 37:2691-2696.

26. Cook A, Laughlin D, Moore M, North D, Wilkins K, Wong G, WallaceScroggs A, Halvorsen L: Differences in glucose values obtained from point-of-care glucose meters and laboratory analysis in critically ill patients. Am J Crit Care 2009, 18:65-71, quiz 72.

27. Karon BS, Gandhi GY, Nuttall GA, Bryant SC, Schaff HV, McMahon MM, Santrach PJ: Accuracy of Roche Accu-chek Inform whole blood capillary, arterial, and venous glucose values in patients receiving intensive intravenous insulin therapy after cardiac surgery. Am J Clin Pathol 2007, 127:919-926.

28. Karon BS, Griesmann L, Scott R, Bryant SC, Dubois JA, Shirey TL, Presti S, Santrach PJ: Evaluation of the impact of hematocrit and other interference on the accuracy of hospital-based glucose meters. Diabetes Technol Ther 2008, 10:111-120.

29. Fekih Hassen M, Ayed S, Gharbi R, Ben Sik Ali H, Marghli S, Elatrous S: Bedside capillary blood glucose measurements in critically ill patients: influence of catecholamine therapy. Diabetes Res Clin Pract 2010, 87:87-91.

30. Finkielman JD, Oyen LJ, Afessa B: Agreement between bedside blood and plasma glucose measurement in the ICU setting. Chest 2005, 127:1749-1751.
31. Lacara T, Domagtoy C, Lickliter D, Quattrocchi K, Snipes L, Kuszaj J, Prasnikar M: Comparison of point-of-care and laboratory glucose analysis in critically ill patients. Am J Crit Care 2007, 16:336-346, quiz 347.

32. Mann EA, Salinas J, Pidcoke HF, Wolf SE, Holcomb JB, Wade CE: Error rates resulting from anemia can be corrected in multiple commonly used point-of-care glucometers. J Trauma 2008, 64:15-20, discussion 20-11.

33. Shearer A, Boehmer M, Closs M, Dela Rosa R, Hamilton J, Horton K McGrath R, Schulman C: Comparison of glucose point-of-care values with laboratory values in critically ill patients. Am J Crit Care 2009, 18:224-230.

34. Ray JG, Hamielec C, Mastracci T: Pilot study of the accuracy of bedside glucometry in the intensive care unit. Crit Care Med 2001, 29:2205-2207.

35. Denfeld QE, Goodell TT, Stafford KN, Kazmierczak S: Precision and accuracy: comparison of point-of-care and laboratory glucose concentrations in cardiothoracic surgery patients. J Cardiovasc Nurs 2011, 26:512-518.

36. Lima AP, Beelen $\mathrm{P}$, Bakker J: Use of a peripheral perfusion index derived from the pulse oximetry signal as a noninvasive indicator of perfusion. Crit Care Med 2002, 30:1210-1213.

37. Rebel A, Rice MA, Fahy BG: Accuracy of point-of-care glucose measurements. J Diabetes Sci Technol 2012, 6:396-411.

38. Yuoh C, Tarek Elghetany M, Petersen JR, Mohammad A, Okorodudu AO: Accuracy and precision of point-of-care testing for glucose and prothrombin time at the critical care units. Clin Chim Acta 2001, 307:119-123.

39. NICE-SUGAR Study Investigators, Finfer S, Liu B, Chittock DR, Norton R, Myburgh JA, McArthur C, Mitchell I, Foster D, Dhingra V, Henderson WR, Ronco JJ, Bellomo R, Cook D, McDonald E, Dodek P, Hébert PC, Heyland DK, Robinson BG: Hypoglycemia and risk of death in critically ill patients. N Engl J Med 2012, 367:1108-1118.

40. Egi M, Bellomo R, Stachowski E, French CJ, Hart GK, Taori G, Hegarty C, Bailey M: Hypoglycemia and outcome in critically ill patients. Mayo Clin Proc 2010, 85:217-224.

41. De Backer D, Creteur J, Preiser JC, Dubois MJ, Vincent JL: Microvascular blood flow is altered in patients with sepsis. Am J Respir Crit Care Med 2002, 166:98-104.

42. Sakr Y, Dubois MJ, De Backer D, Creteur J, Vincent JL: Persistent microcirculatory alterations are associated with organ failure and death in patients with septic shock. Crit Care Med 2004, 32:1825-1831.

doi:10.1186/cc12567

Cite this article as: Inoue et al: Accuracy of blood-glucose

measurements using glucose meters and arterial blood gas analyzers in critically ill adult patients: systematic review. Critical Care 2013 17:R48

\section{Submit your next manuscript to BioMed Central and take full advantage of:}

- Convenient online submission

- Thorough peer review

- No space constraints or color figure charges

- Immediate publication on acceptance

- Inclusion in PubMed, CAS, Scopus and Google Scholar

- Research which is freely available for redistribution

Submit your manuscript at www.biomedcentral.com/submit
C) Biomed Central 